\title{
Nanobiomaterials in dentistry: What's the consequent level
}

"There are two mistakes one can make along the road to truth. Do not go all the way, and not starting."

Buddha

Doing high performance on the dentistry daily practice is a constant challenge for the clinicians and practitioners. Patients' satisfaction, demanding, and aiming long-term oral health-related quality of life has important implications for the clinical practice of dentistry and for the dental scientific field.

Recently, large steps have been done in the field of dental material, and the application of new devices for conservative dentistry, oral surgery, endodontic treatment, and prosthodontics signed the new era of the digital dentistry supported by nanomaterials.

Citing Bhardwaj et al. "The intrigue of nanotechnology comes from the ability to control material properties by assembling such materials at the nanoscale." ${ }^{1,2]}$

Accordingly, with the food and drug administration (FDA) proof and rules, all the materials applied for diagnosis, therapy or investigation should be safe, no allergenic, no toxic, and have a high grade of biocompatibility. The materials used in the biomedical field have two levels of interaction. First, it should be easily accessible, and its macroscopic characteristic should be easy to be manageable. Then, its microscopic, chemical nature should be able to be integrated and to "fit and work" within the body cells and particles. ${ }^{[1-3]}$ However, rebuilding the complex oral environment or replacing the microscopic anatomical features of a tooth in the laboratory is hard to be obtained. Therefore, the nanomaterials data recorded from in vitro investigations and then from clinical trials are significant only when understood within their established endpoints, and can be applied just on specific clinical conditions.

As the time passes, the human knowledge progressed, and the new discoveries in the field of scientific investigation upgraded their bases from millimeter to micrometer and then to nanometer values. Nanotechnology is today defined as the science able to explain and to investigate all the chemical and physical processes happened at the nanoscale level. New discoveries in the field of human physiology and biochemical process made more clear the interaction between cells, tissues, and body organs. In this way, new undiscovered areas of the scientific field called "Nanomaterials" and "Tissue Engineering" started developing new frontiers of investigation and daily discoveries. About tissue engineering, it is not a novelty that the development of new instruments provides the base for creation and application of newer and more advanced materials either "scaffold" either cytokines able to influence stem cells morphogenesis. Consequently, years after year, the application of growth factors for rebuilding bone or soft tissue in the craniofacial district exponentially grew up. It was stated how in the past 10 years $>700,000$ patients have been treated with the application of exogenous cytokines. In the nest future, the use of exogenous cytokines, specifically morphogenetic ones, will become totally safe, common, and the regeneration of hard tissues and soft tissues will likely be carried out as a clinical outpatient procedure. ${ }^{[4]}$

The new understanding of the dental anatomical structure at the level of nanoscale classified the new standards of high-quality dental materials, devices, instruments, and daily practices. Nanodentistry is an ingrowing discipline able to provide new breeding of high-tech excellent clinical devices for the oral health maintenance and treatments. The principal purpose of nanodentistry is to replace the anatomical oral tissue constitution (both soft and hard) by accustoming new biomaterials for enhancing the recovery of damaged tissues due to affection and if requested, for furnishing antibacterial activity. ${ }^{[3,4]}$

Daily dental trade offers numerous FDA certified nanobiomaterials. It may be presented in different macroscopic shapes (liquid, powders, or solid) and structured in several nanostructures (nanotubes, nanoshells, nanopores, nanorods, nanospheres, and nanocapsules) able to replace the microscopic anatomy of the host body.

Recently, Uskokovic stated how "The real reason for the current scientific and technological interest in the physical effects at nanoscale is linked with the 
historic trend of refinement of human knowledge and of the corresponding ability to manipulate with the structural patterns of the Universe" and once again "What we have learnt so far with respect to the peculiarity of physical processes on the nanoscale is only the tip of an iceberg." ${ }^{[5]}$ However, that's a strong definition. Simply the progress goes forward and sometimes, the possibility of "better understanding" the interaction between a material used for doing any therapy in the medical field and not only in dentistry, is undoubtedly welcome.

Marco Cicciù ${ }^{1}$
'Department of Biomedical, Dental Sciences and Morphological
and Functional Images, Dental School, University of Messina,
98100 Messina, Italy

Correspondence: Dr. Marco Cicciù

E-mail: mcicciu@unime.it

\section{REFERENCES}

1. Petersen PE, Bourgeois D, Ogawa H, Estupinan-Day S, Ndiaye C. The global burden of oral diseases and risks to oral health. Bull World Health Organ 2005;83:661-9.
2. Bhardwaj A, Bhardwaj A, Misuriya A, Maroli S, Manjula S, Singh AK, et al. Nanotechnology in dentistry: Present and future. J Int Oral Health 2014;6:121-6.

3. Taniguchi N. Proceedings International Conference on Precision Engineering, Tokyo, Japan; 1974. p. 18-23.

4. Cicciù M. Real opportunity for the present and a forward step for the future of bone tissue engineering. J Craniofac Surg 2017;28:592-3.

5. Uskoković V. Entering the era of nanoscience: Time to be so small. J Biomed Nanotechnol 2013;9:1441-70.

This is an open access journal, and articles are distributed under the terms of the Creative Commons Attribution-NonCommercial-ShareAlike 4.0 License, which allows others to remix, tweak, and build upon the work non-commercially, as long as appropriate credit is given and the new creations are licensed under the identical terms.

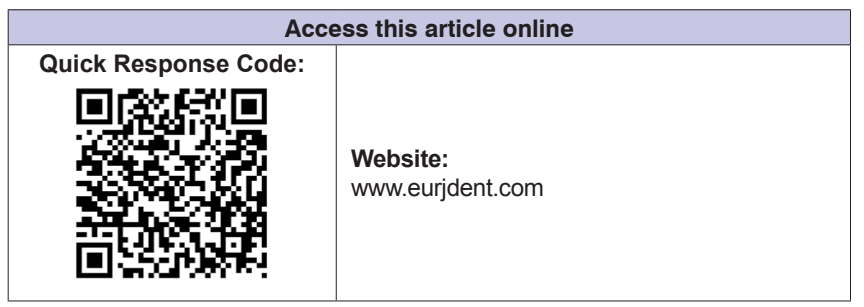

How to cite this article: Cicciù M. Nanobiomaterials in dentistry: What's the consequent level. Eur J Dent 2018;12:161-2.

DOI: 10.4103/ejd.ejd_69_18 